Research Article

\title{
Study on a Class of Piecewise Nonlinear Systems with Fractional Delay
}

\author{
Meiqi Wang $\mathbb{D}^{1,2}$ Wenli Ma, ${ }^{1,2}$ Enli Chen $\mathbb{D}^{1,2}$ and Yujian Chang ${ }^{1,3}$ \\ ${ }^{1}$ State Key Laboratory of Mechanical Behavior and System Safety of Traffic Engineering Structures, \\ Shijiazhuang Tiedao University, Shijiazhuang 050043, China \\ ${ }^{2}$ School of Mechanical Engineering, Shijiazhuang Tiedao University, Shijiazhuang 050043, China \\ ${ }^{3}$ School of Electrical and Electronic Engineering, Shijiazhuang Tiedao University, Shijiazhuang 050043, China \\ Correspondence should be addressed to Enli Chen; chenenlistdu@163.com
}

Received 8 June 2021; Revised 6 August 2021; Accepted 7 August 2021; Published 7 October 2021

Academic Editor: Felix Albu

Copyright (c) 2021 Meiqi Wang et al. This is an open access article distributed under the Creative Commons Attribution License, which permits unrestricted use, distribution, and reproduction in any medium, provided the original work is properly cited.

\begin{abstract}
In this paper, a dynamic model of piecewise nonlinear system with fractional-order time delay is simplified. The amplitude frequency response equation of the dynamic model of piecewise nonlinear system with fractional-order time delay under periodic excitation is obtained by using the average method. It is found that the amplitude of the system changes when the external excitation frequency changes. At the same time, the amplitude frequency response characteristics of the system under different time delay parameters, different fractional-order parameters, and coefficient are studied. By analyzing the amplitude frequency response characteristics, the influence of time delay and fractional-order parameters on the stability of the system is analyzed in this paper, and the bifurcation equations of the system are studied by using the theory of continuity. The transition sets under different piecewise states and the constrained bifurcation behaviors under the corresponding unfolding parameters are obtained. The variation of the bifurcation topology of the system with the change of system parameters is given.
\end{abstract}

\section{Introduction}

The existence of time delay behavior is often considered as the root of complex behavior of dynamic system. In the system modeling, researchers have studied the periodic motion and dynamic response of nonlinear systems with fractional differential delay. Jia and Jiang [1] studied the synchronization of fractional time-delay chaotic systems and their application in secure communication. Yang and Liu [2] studied the main resonance of fractional delay Duffing system. The effects of high frequency signal, fractional damping, and delay parameters on vibration resonance are obtained by theoretical and numerical simulation. Wen and Shen [3] studied the influence of fractional delay feedback on the dynamic characteristics of Duffing system. Zhang et al. [4] gave a new criterion for oscillation of second-order forced mixed nonlinear differential equations by using Riccati transformation. Duan [5] studied the global dynamics of a predator-prey model. Because the predator behavior has a lag effect on the change of its number, the model is a system of delay differential equations. Finally, the corresponding numerical simulation is given for the theoretical results. Li et al. [6] studied the control synchronization problem of coronary artery system with input delay and disturbance. Chai [7] studied the parameter identification of a class of general time-delay chaotic systems with unknown and time-varying parameters.

So far, fractional calculus has been widely concerned by researchers in different fields. It has been widely studied in the fields of textile [8], electricity [9], automatic control theory [10], signal engineering [11], and mechanics [12]. Many chaotic behaviors have been found in fractional-order systems. In engineering design and application, in order to make rational use of the dynamic characteristics of nonlinear system with fractional calculus and avoid its adverse effects on the system, researchers have carried out a lot of related research. Ozkan et al. [13] constructed the exact solutions of nonlinear space-time fractional KP-BBM and obtained 
different types of exact solutions. Chang et al. [14] studied $1+n$-dimensional time-fractional partial differential equations. Two special forms of nonlinear time-fractional diffusion convection equations are studied by using the Lie group analysis method. The invariant solutions and some exact solutions are obtained. Liu et al. [15] proposed a numerical method to study fractional-order nonlinear systems and studied the chaotic set in the system. Shen et al. [16] studied the dynamic phenomenon of resonance of Duffing oscillator with fractional order and analyzed the influence of some parameters on the system. The dynamic characteristics of the system are obtained through simulation experiments.

With the rapid development of nonlinear theory and the gradual deepening of the research on piecewise problems, the research on nonlinear problems with fractional calculus and piecewise nonlinear problems is more common. Parisa et al. [17] proposed a new method for solving fractional differential equations and gave the error bounds in the sense of Sobolev norm. The numerical results show the effectiveness of the method. Geng et al. [18] studied the truncated Euler-Maruyama (EM) method for stochastic differential equations with piecewise continuous arguments and considered the strong convergence theory under local Lipschitz condition and Khasminskii-type condition. Martin and Basu [19] analyzed the three-wave and four-wave resonances of capillary gravity water waves on the free surface of water flow with piecewise equal vorticity. Ramirez and Alves [20] studied the bifurcation limit cycles of piecewise nonHamiltonian systems with nonlinear switching manifolds. Liu Fei et al. [21] studied the cyclic response characteristics of a class of piecewise nonlinear elastic damped double constraint systems. Yang et al. [22] studied a class of piecewise smooth integrable non-Hamiltonian systems with centers. Yu [23] used the Melnikov function method to study the maximum number of limit cycles bifurcated from the periodic cycle domain of the nonlinear center of a class of discontinuous generalized Lienard differential systems.

To sum up, the research on nonlinear systems mainly includes nonlinear systems with fractional-order delay and piecewise nonlinear systems with fractional-order delay, while the research on piecewise nonlinear systems with fractional-order delay is rare. In this paper, the dynamic model of a piecewise nonlinear system with fractional-order time delay is studied. The amplitude frequency response equation of the system under periodic excitation is obtained by using the averaging method. The amplitude frequency response characteristics under different time delay parameters and fractional-order parameters are discussed. The influence of time delay and fractional-order term on the stability of the system is analyzed.

\section{Approximate Analytical Solution}

In engineering design and application, there are often piecewise nonlinear systems with fractional delay. The magnetorheological damper is taken as an example; a magnetorheological damper is composed of piston, cylinder, magnetorheological fluid, damping-elastic part, and rubber reset element. When subjected to shear load, the rubber and magnetorheological damper play a major role in damping. Rubber is a typical viscoelastic solid, and the fractional-order model can be used to describe the force of this part. Magnetic fluid also has viscoelastic properties, which can be described by the fractional constitutive equation. Therefore, the magnetorheological damper can be simplified as a dynamic model of a class of piecewise nonlinear systems with fractional delay shown in Figure 1.

The dynamic equation of the system is shown as follows:

$$
\begin{aligned}
& m \ddot{x}(t)+k_{1} x(t)+c \dot{x}(t)+k_{2} x(t)^{3}+ \\
& y[x(t), \dot{x}(t)]+K D^{p}[x(t-\tau)]=F \cos (\omega t),
\end{aligned}
$$

where $x$ is the vibration displacement of the mass, $F \cos (\omega t)$ is the periodic external excitation, and $y[x(t), \dot{x}(t)]$ is the piecewise nonlinear binding force:

$$
y[x(t), \dot{x}(t)]=y_{\text {buf }}[x(t), \dot{x}(t)]-c \dot{x}(t)-k_{2} x(t)^{3},
$$

where

$$
\begin{aligned}
& y_{\text {buf }}[x(t), \dot{x}(t)]= \\
& \begin{cases}k_{2} x(t)^{3}+c \dot{x}(t), & -d_{1}<x<d_{1}, \\
\left(k_{1}\left(x(t)-d_{1}\right)+2 c \dot{x}(t)+k_{2}\left(x(t)-d_{1}\right)^{3}+k_{2} x(t)^{3}\right), & d_{1} \leq x \leq d_{2}, \\
\left(k_{1}\left(x(t)+d_{1}\right)+2 c \dot{x}(t)+k_{2}\left(x(t)+d_{1}\right)^{3}+k_{2} x(t)^{3}\right), & -d_{2} \leq x \leq-d_{1}, \\
\left(k_{1}\left(x(t)-d_{1}\right)+2 c \dot{x}(t)+k_{2}\left(x(t)-d_{1}\right)^{3}+k_{3}\left(x(t)-d_{2}\right)+k_{2} x(t)^{3}\right), & d_{2}<x, \\
\left(k_{1}\left(x(t)+d_{1}\right)+2 c \dot{x}(t)+k_{2}\left(x(t)+d_{1}\right)^{3}+k_{3}\left(x(t)+d_{2}\right)+k_{2} x(t)^{3}\right), & x<-d_{2} .\end{cases}
\end{aligned}
$$

In equation (1), $m$ is the mass of the system, $k_{1}$ is the linear stiffness coefficient, $k_{2}$ is the nonlinear stiffness coefficient, $c$ is the linear viscous damping coefficient, $F$ is the excitation amplitude, $\omega$ is the excitation frequency, $\operatorname{KD}_{t}^{p}[x(t-\tau)]$ is the derivative of $x(t)$ to $t, K(K>0)$ is the coefficient of the fractional term, $p(0<p<1)$ is the order of the fractional term, $\tau$ is the delay coefficient, and $d_{1}$ and $d_{2}$ are different segment positions.

The fractional term is defined by Caputo as follows: 


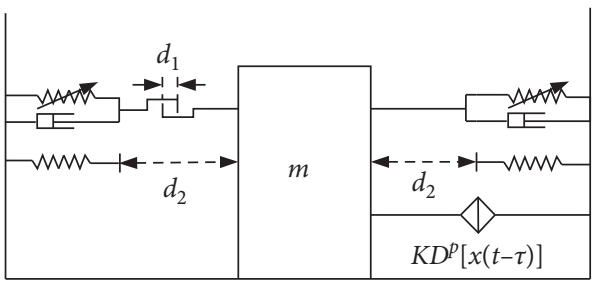

The dynamic equation of the system is transformed to obtain the following result:

Figure 1: Nonlinear systems with piecewise time delay.

$$
D^{p}[x(t)]=\frac{1}{\Gamma(1-p)} \int_{0}^{t} \frac{x(u)}{(t-u)} \mathrm{d} u
$$

$$
\begin{aligned}
& \ddot{x}(t)+\omega_{0}^{2} x(t)+\varepsilon g[x(t), \dot{x}(t)]+\varepsilon \mu D^{p}[x(t-\tau)]=\varepsilon f \cos (\omega t), \\
& \varepsilon g[x(t), \dot{x}(t)]= \\
& \begin{cases}\varepsilon \alpha \dot{x}(t)+\varepsilon \beta_{2} x(t)^{3}, & -d_{1}<x<d_{1}, \\
\left(\varepsilon \beta_{1}\left(x(t)-d_{1}\right)+2 \varepsilon \alpha \dot{x}(t)+\varepsilon \beta_{2}\left(x(t)-d_{1}\right)^{3}+\varepsilon \beta_{2} x(t)^{3}\right), & d_{1} \leq x \leq d_{2}, \\
\left(\varepsilon \beta_{1}\left(x(t)+d_{1}\right)+2 \varepsilon \alpha \dot{x}(t)+\varepsilon \beta_{2}\left(x(t)+d_{1}\right)^{3}+\varepsilon \beta_{2} x(t)^{3}\right), & -d_{2} \leq x \leq-d_{1}, \\
\left(\varepsilon \beta_{1}\left(x(t)-d_{1}\right)+\varepsilon \beta_{2}\left(x(t)-d_{1}\right)^{3}+\varepsilon \beta_{3}\left(x(t)-d_{2}\right)+\varepsilon \beta_{2} x(t)^{3}+2 \varepsilon \alpha \dot{x}(t)\right), & d_{2}<x, \\
\left(\varepsilon \beta_{1}\left(x(t)+d_{1}\right)+\varepsilon \beta_{2}\left(x(t)+d_{1}\right)^{3}+\varepsilon \beta_{3}\left(x(t)+d_{2}\right)+\varepsilon \beta_{2} x(t)^{3}+2 \varepsilon \alpha \dot{x}(t)\right), & x<-d_{2} .\end{cases}
\end{aligned}
$$

In equations (5) and (6), $\omega_{0}=\sqrt{k_{1} / m}$, where $\omega_{0}$ are the natural frequencies of the system, $\varepsilon \alpha=c / m, \varepsilon \beta_{1}=k_{1} / m$, $\varepsilon \beta_{2}=k_{2} / m, \quad \varepsilon \beta_{3}=k_{3} / m, \quad \varepsilon \mu=K / m, \quad \varepsilon f=F / m, \quad$ and $\varepsilon g[x(t), \dot{x}(t)]=y_{\text {buf }}[x(t), \dot{x}(t)]$, where $\varepsilon$ is a number greater than 0 and far less than 1 , so it meets the requirements of approximate solution.

The average method is used to solve equation (5), which mainly studies the main resonance of the system, namely, $\omega^{2}=\omega_{0}^{2}+\varepsilon \sigma$, where $\sigma$ is the tuning parameter.

$$
\ddot{x}(t)+\omega^{2} x(t)=\varepsilon\left\{f \cos (\omega t)-g[x(t), \dot{x}(t)]-\mu D^{p}[x(t-\tau)]+\sigma x(t)\right\} .
$$

When $\sigma=0$, the derived system of equation (7) is a linear conservative system.

$$
\ddot{x}(t)+\omega^{2} x(t)=0 .
$$

The free vibration solution of the derived system is

$$
\begin{aligned}
x(t) & =a \cos (\omega t+\theta), \\
\dot{x}(t) & =-a \omega \sin (\omega t+\theta), \\
\dot{x}(t-\tau) & =-a \omega \sin (\omega(t-\tau)+\theta),
\end{aligned}
$$

where $a$ and $\theta$ are functions of time $t$, respectively. Let $\varphi=\omega t+\theta, d_{1}=a \cos \varphi_{1}$, and $d_{2}=a \cos \varphi_{2}$.

Considering the change of $a$ and $\theta$, the differential equation (9) is derived by eliminating the following equation:

$$
\dot{a} \cos \varphi+a \dot{\theta} \sin \varphi=0 .
$$

Differentiating equation (10) with respect to time and substituting it into equation (7), we obtain

$$
-\dot{a} \sin \varphi+a \dot{\theta} \cos \varphi=\frac{\varepsilon}{\omega}\left\{f \cos (\omega t)-g[x(t), \dot{x}(t)]-\mu D^{p}[x(t-\tau)]+\sigma x(t)\right\} .
$$

From equations (10) and (11), we obtain 


$$
\begin{gathered}
\dot{a}=-\frac{1}{\omega}\left[P_{1}(a, \theta)+P_{2}(a, \theta)+P_{3}(a, \theta, \tau)\right] \sin \varphi, \\
\dot{a}=\frac{1}{\omega}\left[P_{1}(a, \theta)+P_{2}(a, \theta)+P_{3}(a, \theta, \tau)\right] \cos \varphi,
\end{gathered}
$$

where

$$
\begin{aligned}
& P_{1}(a, \theta)=\varepsilon[f \cos (\omega t)+\sigma a \cos \varphi], \\
& P_{2}(a, \theta)=-\varepsilon g[a \cos \varphi,-a \sin \varphi], \\
& P_{3}(a, \theta)=-\varepsilon \mu D^{p}[a \cos (\varphi-\omega \tau)] .
\end{aligned}
$$

When $\varepsilon$ is sufficiently small, $a$ and $\theta$ are functions that change slowly around the constant. The term to the right of the equal sign in equation (12) is approximately replaced by the average value in one period of $\varphi$, and it is considered that $a$ and $\theta$ remain unchanged in one period of $\varphi$. The integral of (12) on $[0, t]$ is obtained as follows:

$$
\begin{aligned}
\dot{a}= & -\frac{1}{\omega T} \int_{0}^{T} P_{1}(a, \theta) \sin \varphi \mathrm{d} \varphi-\frac{1}{\omega T} \int_{0}^{T} P_{2}(a, \theta) \sin \varphi \mathrm{d} \varphi \\
& -\frac{1}{\omega T} \int_{0}^{T} P_{3}(a, \theta, \tau) \sin \varphi \mathrm{d} \varphi=\dot{a}_{1}+\dot{a}_{2}+\dot{a}_{3}, \\
a \dot{\theta}= & \frac{1}{\omega T} \int_{0}^{T} P_{1}(a, \theta) \cos \varphi \mathrm{d} \varphi+\frac{1}{\omega T} \int_{0}^{T} P_{2}(a, \theta) \sin \varphi \mathrm{d} \varphi \\
& +\frac{1}{\omega T} \int_{0}^{T} P_{3}(a, \theta, \tau) \cos \varphi \mathrm{d} \varphi=a \dot{\theta}_{1}+a \dot{\theta}_{2}+a \dot{\theta}_{3} .
\end{aligned}
$$

When the integrand is a periodic function, $T=2 \pi$; when the integrand is an aperiodic function, $T=\infty$. The results of (14) are as follows:

$$
\begin{aligned}
\dot{a}_{1} & =-\frac{1}{\omega 2 \pi} \int_{0}^{2 \pi} \varepsilon[f \cos (\omega t)+\sigma a \cos \varphi] \sin \varphi \mathrm{d} \varphi \\
& =-\frac{\varepsilon f \sin \theta}{2 \omega}, \\
a \dot{\theta}_{1} & =-\frac{1}{\omega 2 \pi} \int_{0}^{2 \pi} \varepsilon[f \cos (\omega t)+\sigma a \cos \varphi] \cos \varphi \mathrm{d} \varphi \\
& =\frac{\varepsilon}{2 \omega}(\sigma a+f \cos \theta) .
\end{aligned}
$$
obtain

$$
\begin{aligned}
D_{t}^{p} \sin (\omega t) & =\omega^{p} \sin \left(\omega t+\frac{\alpha \pi}{2}\right) \\
D_{t}^{p} \cos (\omega t) & =\omega^{p} \cos \left(\omega t+\frac{\alpha \pi}{2}\right) \\
\dot{a}_{3} & =-\lim _{T \longrightarrow \infty} \frac{1}{\omega T} \int_{0}^{T} P_{2}(a, \theta, \tau) \sin \varphi \mathrm{d} \varphi \\
& =\lim _{T \longrightarrow \infty} \frac{\varepsilon \mu}{\omega T} \int_{0}^{T} D^{p}[a \cos (\varphi-\omega \tau)] \sin \varphi \mathrm{d} \varphi \\
& =\frac{\varepsilon \mu a \omega^{p-1}}{2} \sin \left(\frac{p \pi}{2}-\omega \tau\right), \\
\dot{\theta}_{3} & =-\lim _{T \longrightarrow \infty} \frac{1}{\omega T} \int_{0}^{T} P_{2}(a, \theta, \tau) \cos \varphi \mathrm{d} \varphi \\
& =\lim _{T \longrightarrow \infty} \frac{\varepsilon \mu}{\omega T} \int_{0}^{T}-D^{p}[a \cos (\varphi-\omega \tau)] \cos \varphi \mathrm{d} \varphi \\
& =-\frac{\varepsilon \mu a \omega}{2-1} \cos \left(\frac{p \pi}{2}-\omega \tau\right) .
\end{aligned}
$$

Next, we calculate $\dot{a}_{2}$ and $a \dot{\theta}_{2}$ :

$$
\begin{aligned}
\dot{a}_{2} & =-\frac{1}{\omega 2 \pi} \int_{0}^{2 \pi}-\varepsilon g(a \cos \varphi,-a \omega \sin \varphi) \sin \varphi \mathrm{d} \varphi \\
& =\Phi(a, \omega), \\
a \dot{\theta}_{2} & =\frac{1}{\omega 2 \pi} \int_{0}^{2 \pi}-\varepsilon g(a \cos \varphi,-a \omega \sin \varphi) \cos \varphi \mathrm{d} \varphi \\
& =\Lambda(a, \omega) .
\end{aligned}
$$

In conclusion,

$$
\begin{aligned}
& \dot{a}=-\frac{\varepsilon f \sin \theta}{2 \omega}+\Phi(a, \omega)+\frac{\varepsilon \mu a \omega^{p-1}}{2} \sin \left(\frac{p \pi}{2}-\omega \tau\right), \\
& \dot{a}=\frac{\varepsilon}{2 \omega}(\sigma a+f \cos \theta)+\Lambda(a, \omega)-\frac{\varepsilon \mu a \omega^{p-1}}{2} \cos \left(\frac{p \pi}{2}-\omega \tau\right) .
\end{aligned}
$$

Equation (18) is the approximate analytical solution of the system.

Let $\dot{a}=0$ and $\dot{\theta}=0$ study the steady-state solution of the system: 


$$
\begin{aligned}
& 0=-\frac{F \sin \theta}{2 \omega m}+\Phi(a, \omega)+\frac{K a \omega^{p-1}}{2 m} \sin \left(\frac{p \pi}{2}-\omega \tau\right), \\
& 0=\frac{a}{2 \omega}-\frac{k_{1} a}{2 \omega m}+\frac{F \cos \theta}{2 \omega m}+\Lambda(a, \omega)-\frac{K a \omega^{p-1}}{2 m} \cos \left(\frac{p \pi}{2}-\omega \tau\right) .
\end{aligned}
$$

When the magnitude and frequency of the excitation are determined, the phase trajectory in the moving phase plane can be determined by equation (19). When the numerator and denominator of equation (19) are zero at the same time, the singularity $\left(a_{s}, \theta_{s}\right)$ corresponding to the steady-state response of the system can be obtained, and the amplitude frequency characteristics of the system under the action of external periodic excitation can be derived by eliminating $\theta_{s}$.

$$
\begin{aligned}
& {\left[2 \omega m \Phi(a, \omega)+K a \omega^{p} \sin \left(\frac{p \pi}{2}-\omega \tau\right)\right]^{2}} \\
& \quad+\left[-k_{1} a+m \omega^{2} a+2 \omega m \Lambda(a, \omega)-K a \omega^{p} \cos \left(\frac{p \pi}{2}-\omega \tau\right)\right]^{2}=F^{2} .
\end{aligned}
$$

\section{Study on Periodic Response Characteristics of Nonlinear System}

Select a set of system parameters: $m=1, k_{1}=1, k_{2}=1$, $k_{3}=50, c=0.15, K_{1}=0.5, p=0.2, d_{1}=0.2, d_{2}=1, F=1$, and $\tau=0.1$. According to equation (20), the amplitude frequency relationship of the system can be analyzed.

As can be seen from Figure 2, when the external excitation frequency changes, the amplitude of the system changes. The amplitude frequency curve presents a smooth curve at $d_{1}=0.2$. At the segment point $d_{2}=1$, there is a break and a jump phenomenon. After passing through the critical position $d_{2}=1$, it is smooth again.

Figure 3 shows that the response amplitude of the timedelayed system lags behind that of the system without time delay. The increase of fractional delay term increases the response time of the system, resulting in the delay of amplitude frequency response of the system.

3.1. Time Delay Analysis. Change the value of system delay, and then analyze the change of amplitude frequency response curve. The result of amplitude frequency response curve is shown in Figure 4.

It can be seen from Figure 4 that when the time delay of the system is small (as shown in Figures 4(a) and 4(b)), with the increase of time delay, the amplitude of amplitude frequency response curve gradually increases and the degree of nonlinear bending increases. When the time delay of the system is large (as shown in Figures 4(c) and 4(d)), the topological structure of the amplitude frequency response curve will change. At the segment point $d_{2}=1$, the degree of refraction increases and an independent loop appears in the high frequency region. With the increase of time delay, the number of independent loops in the same frequency range increases.

3.2. Analysis of the Variation of Coefficients and Orders of Fractional Differential Terms. When the delay parameter is small, the influence of the coefficient and order of the

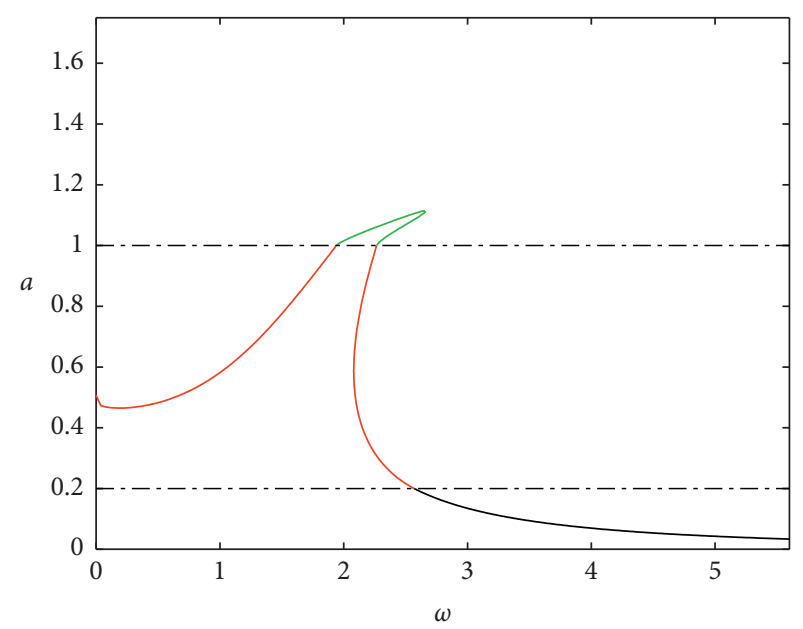

Figure 2: System amplitude frequency response curve and its partial enlargement.

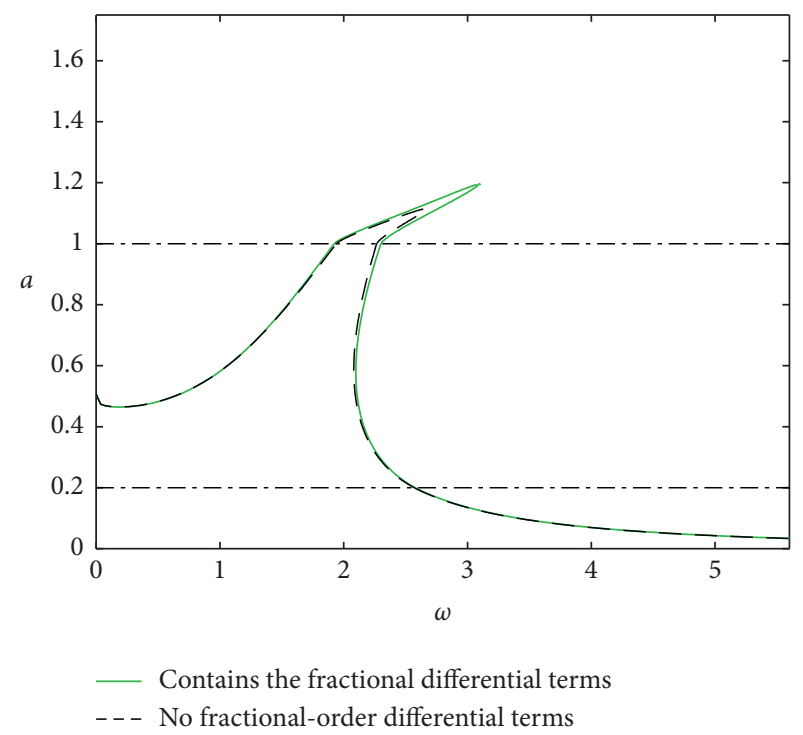

FIgUre 3: Comparison diagram of amplitude frequency response curves of systems with and without time delay and its local enlargement diagram.

fractional differential term on the amplitude frequency response curve of the system is shown in Figures 5 and 6.

According to Figure 5, the amplitude frequency response curve of the system changes obviously with the change of the order of the fractional differential term. When the fractional order is small, the fractional-order term is close to the linear stiffness term. However, with the increase of the order of the fractional differential term, the fractional term gradually turns into a linear damping term. Therefore, with the increase of the order of the fractional differential term, the peak value of the amplitude frequency response curve gradually decreases and the jump at the segment point $d_{2}=$ 1 gradually disappears.

It can be seen from Figure 6 that when $K_{1}$ takes a fixed value, the order $p$ of the fractional differential term has a great influence on the amplitude-frequency response curve. When $p$ 


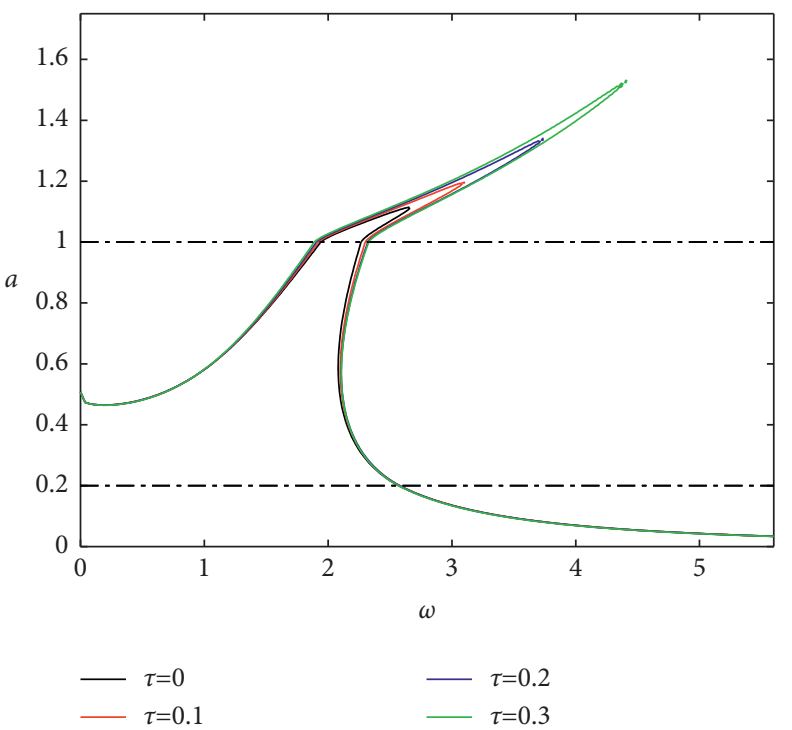

(a)

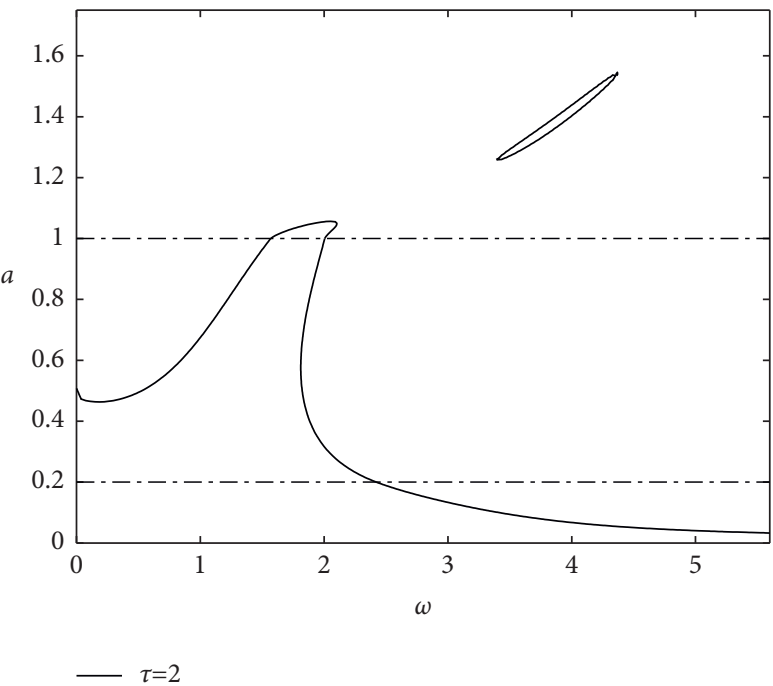

(c)

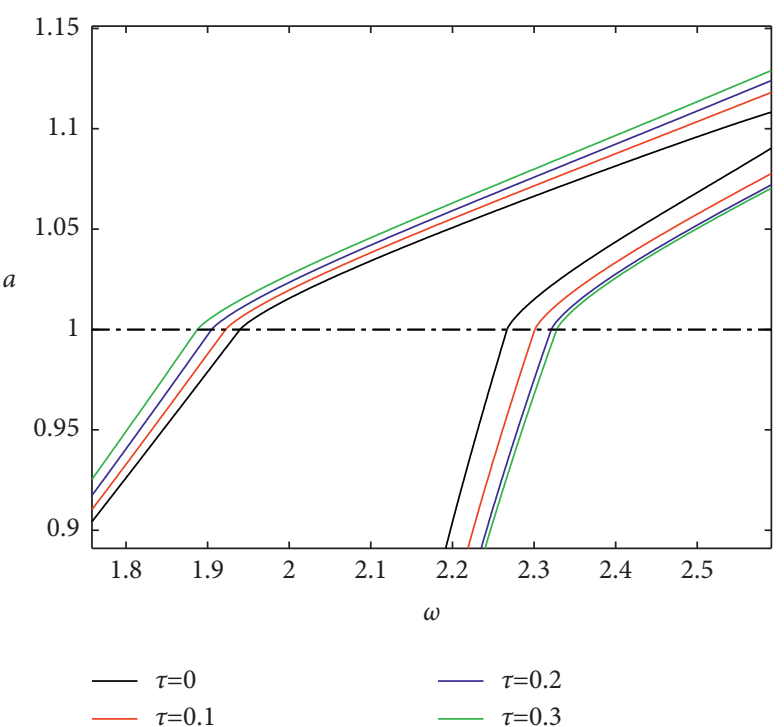

(b)

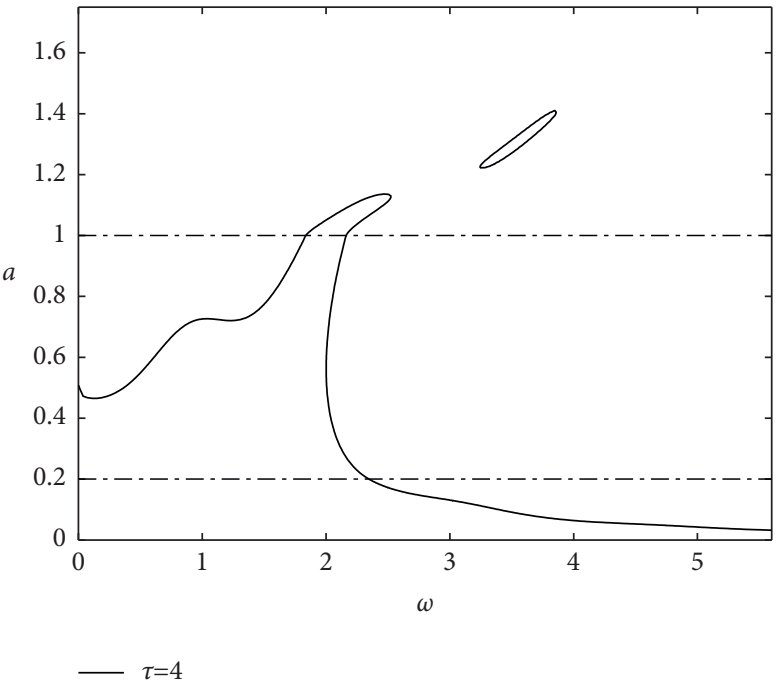

(d)

Figure 4: Amplitude frequency response curve with time lag and its partial enlargement. (a) The amplitude frequency response curve changes with time delay ( $\tau=0, \tau=0.1, \tau=0.2$, and $\tau=0.3$ ). (b) Partial enlarged drawing $(\tau=0, \tau=0.1, \tau=0.2, \tau=0.3)$. (c) The amplitude frequency response curve changes with time delay $(\tau=2)$. (d) The amplitude frequency response curve changes with time delay $(\tau=4)$.

is small, the peak value of the amplitude-frequency response curve increases with the increase of the order coefficient $K_{1}$ of the fractional differential term. At the segmentation point $d_{2}=$ 1 , the deformation gradually increases, and the bending degree of the curve at the segmentation point $d_{1}=0.2$ gradually increases. When $p$ is larger, the peak value of the amplitudefrequency curve decreases with the increase of the fractional differential coefficient $K_{1}$. At the segmentation point $d_{2}=1$, the degree of deformation decreases or even completely disappears.

\section{Bifurcation Response Characteristics}

According to the singularity theory, the transition set divides the unfolding parameter space into several subregions. The bifurcation behavior in each region is persistent. The bifurcation diagram is topologically equivalent, but the system response has different robustness with the change of unfolding parameters. The bifurcation behavior on the transfer set is nonpersistent and is in the critical state of bifurcation topology change.

From the amplitude frequency equation of the system, the bifurcation equation of the system under three constraint conditions can be obtained.

(1) When $0<a<d_{1}$,

$$
\begin{aligned}
& \Lambda(a, \omega)=-\frac{3}{4} k_{2} a^{3}, \\
& \Phi(a, \omega)=c \omega a .
\end{aligned}
$$

The bifurcation equation of the system is as follows: 


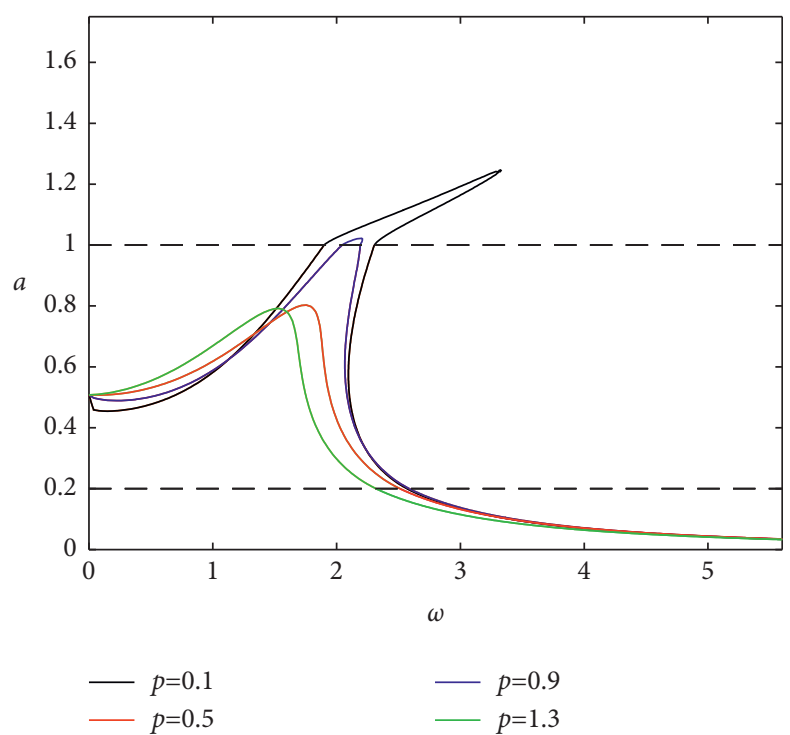

Figure 5: Amplitude frequency response curve with the order of fractional differential term.

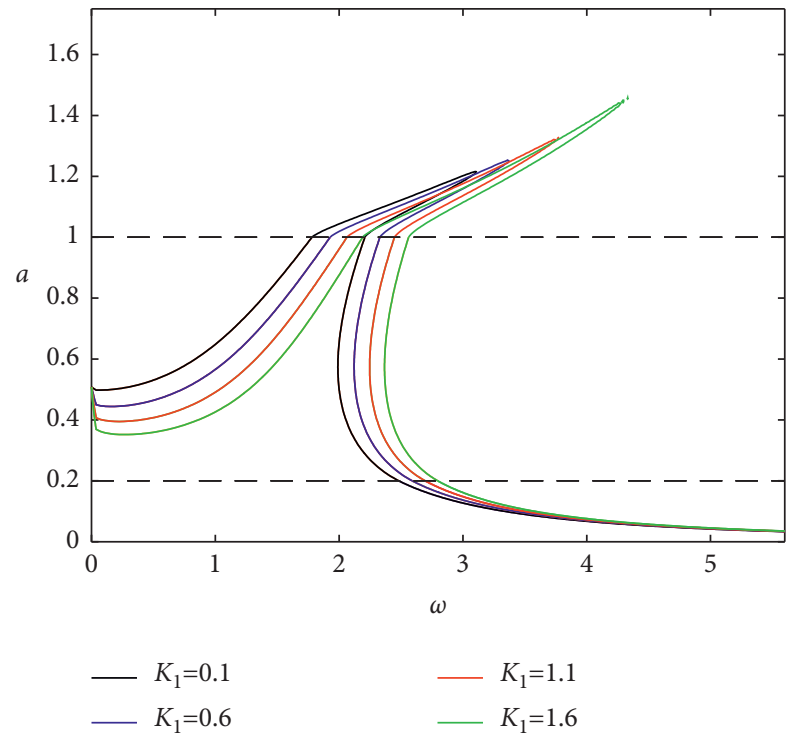

(a)

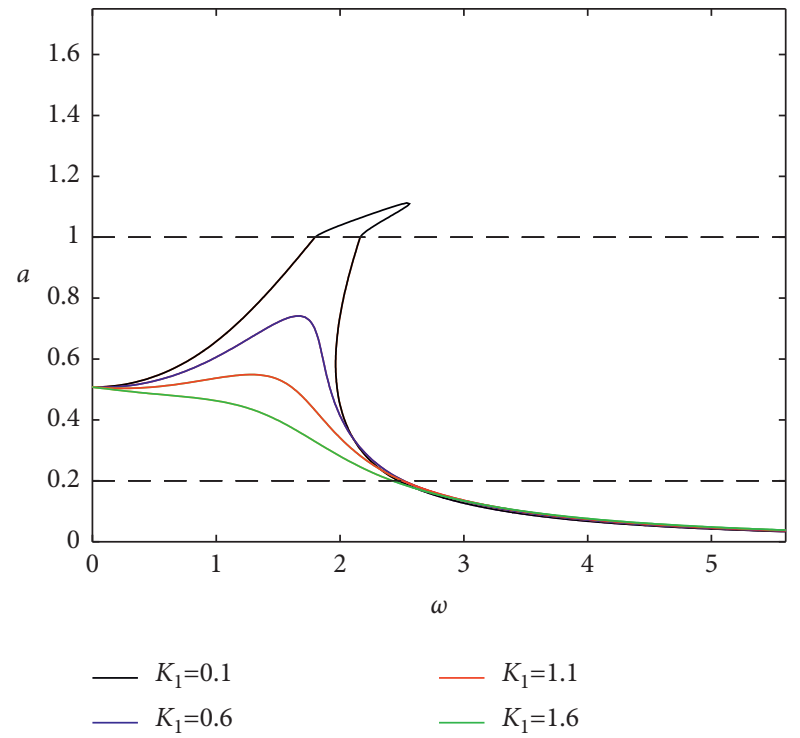

(b)

Figure 6: Amplitude frequency response curve with the coefficient of fractional differential term: (a) $p=0.1$ and (b) $p=0.9$.

$$
a^{6}+\chi_{1} a^{4}+\chi_{2} a^{2}+b=0
$$

where $\chi_{1}=\lambda_{1} / \lambda, \chi_{2}=\lambda_{2} / \lambda$, and $b=\lambda_{3} / \lambda$, in which

$$
\begin{aligned}
& \lambda=\frac{9}{4} k_{2}^{2} m^{2} \omega^{2}, \\
& \lambda_{1}=3 k_{2} m \omega\left(k_{1}-m \omega^{2}+K \omega^{p} \cos \left(\frac{p \pi}{2}-\tau \omega\right)\right), \\
& \lambda_{2}=\left(2 c m \omega^{2}+K \omega^{p} \sin \left(\frac{p \pi}{2}-\tau \omega\right)\right)^{2}, \\
& \lambda_{3}=\left(k_{1}-m \omega^{2}+K \omega^{p} \cos \left(\frac{p \pi}{2}-\tau \omega\right)\right)^{2}-F^{2} .
\end{aligned}
$$

Equation (22) is the universal unfolding of normal form $a^{6}+b=0$. The codimension is 2 . Take $b$ as bifurcation parameter and $\chi_{1}$ and $\chi_{2}$ as unfolding parameter. The transition set is composed of bifurcation point set, delay point set, and hyperbolic limit point set.

The set of bifurcation points is empty.

The delay point set is calculated as $H_{R}=\left\{3 \chi_{2}^{2} / \chi_{1}^{2}-\chi_{2}=0\right\}$ and $H_{I}=\left\{\chi_{1} d_{1}^{2}+2 \chi_{2}=0\right\}$.

The hyperbolic limit point set is calculated as $D L_{R}=$ $\left\{4 \chi_{2}^{2} / \chi_{1}^{2}-\chi_{2}=0\right\}$ and $D L_{I}=\left\{d_{1}^{1}+\chi_{1} d_{1}^{2}+\chi_{2}=0\right\}$.

The migration set is $\Sigma=B \cup H_{R} \cup H_{I} \cup D L_{R} \cup D L_{I}$. The transition set and bifurcation diagram are shown in Figure 7.

By calculation, the bifurcation point set of the system is an empty set, so the transition set includes the time delay set 


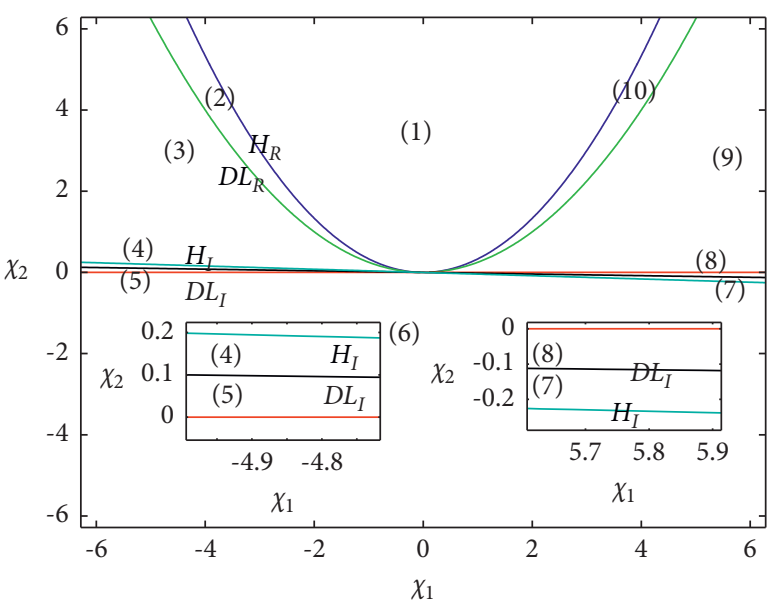

(a)
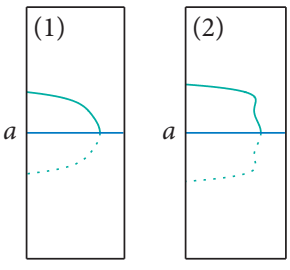

$b$

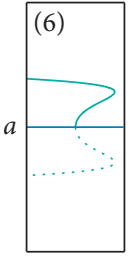

$b$

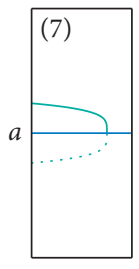

$b$

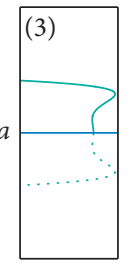

$b$

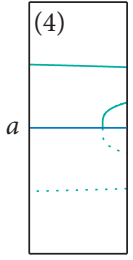

$b$

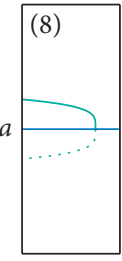

$b$

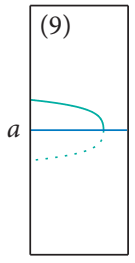

$b$

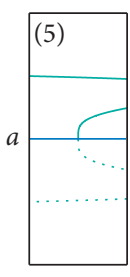

$b$

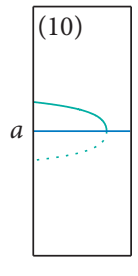

(b)

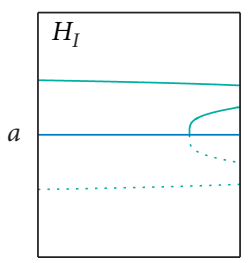

$b$

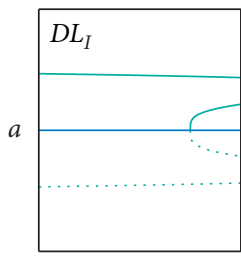

$b$
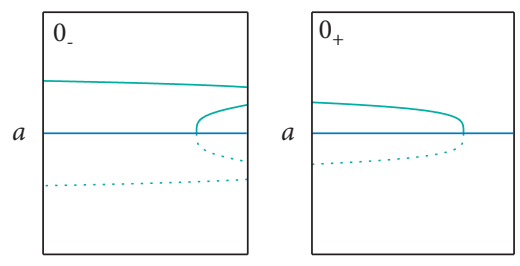

$b$

(c)

Figure 7: (a) Transition set and (b, c) bifurcation diagrams of the system.

and hyperbolic limit point set. As shown in Figure 7(a), the transition set divides the plane into 10 regions. In order to facilitate observation, the fine part of the transition set is locally enlarged. The bifurcation diagrams corresponding to each region and the transition set are shown in Figures 7(b) and $7(\mathrm{c})$, and the bifurcation diagrams are abundant.
Different bifurcation modes reflect the dynamic behavior of the system without any parameters, so when $0<a<d_{1}$, the dynamic behavior can be controlled by changing the parameters $\chi_{1}$ and $\chi_{2}$.

(2) When $d_{1} \leq a \leq d_{2}$,

$$
\begin{aligned}
& \Lambda(a, \omega)=-\frac{1}{4 \pi}\left(3 k_{2} \pi a^{3}+18 k_{2} \varphi_{1} a^{3}-4 k_{1} a \sin 2 \varphi_{1}+8 k_{1} \varphi_{1} a-14 k_{2} a^{3} \sin 2 \varphi_{1}-\frac{1}{2} k_{2} a^{3} \sin 4 \varphi_{1}+12 k_{2} \varphi_{1} a^{3} \cos 2 \varphi_{1}\right), \\
& \Phi(a, \omega)=\frac{1}{\pi}\left(2 c \omega a \varphi_{1}-c \omega a \sin 2 \varphi_{1}+c \omega \pi a\right) . \\
& \text { The bifurcation equation of the system is }
\end{aligned}
$$


where $\chi_{1}^{\prime}=\lambda_{1}^{\prime} / \lambda^{\prime}, \chi_{2}^{\prime}=\lambda_{2}^{\prime} / \lambda^{\prime}$, and $b^{\prime}=\lambda_{3}^{\prime} / \lambda^{\prime}$, in which

$$
\begin{aligned}
\lambda^{\prime}= & 4 m^{2} \omega^{2}\left\{\left(0.75 k_{2}+1.43 \varphi_{1} k_{2}-1.12 k_{2} \sin 2 \varphi_{1}+0.95 \varphi_{1} k_{2} \cos 2 \varphi_{1}\right)^{2}\right\}, \\
\lambda_{1}^{\prime}= & 4 m \omega\left(0.75 k_{2}+1.43 \varphi_{1} k_{2}-1.12 k_{2} \sin 2 \varphi_{1}+0.95 \varphi_{1} k_{2} \cos 2 \varphi_{1}\right)\left(k 1-1 m \omega^{2}+K \omega^{p} \cos \left(\frac{p \pi}{2}-\tau \omega\right)\right. \\
& \left.-2 m \omega\left(0.32 k_{1} \sin 2 \varphi_{2}-0.64 \varphi_{1} k_{1}+0.12 k_{2} \sin 4 \varphi_{1}\right)\right), \\
\lambda_{2}^{\prime}= & \left(k_{1}-m \omega^{2}+K \omega^{p} \cos \left(\frac{p \pi}{2}-\tau \omega\right)-2 m \omega\left(0.32 k_{1} \sin 2 \varphi_{2}-0.64 \varphi_{1} k_{1}+0.12 k_{2} \sin 4 \varphi_{1}\right)\right)^{2} \\
& +\left(2 m \omega\left(c \omega+0.64 c \varphi_{1} \omega-0.32 c \omega \sin \left(2 \varphi_{1}\right)\right)+K \omega^{p} \sin \left(\frac{p \pi}{2}-\tau \omega\right)\right)^{2}, \\
\lambda_{3}^{\prime}= & -F^{2} .
\end{aligned}
$$

It is similar to Case 1, but they still have different bifurcation characteristics due to different unfolding parameters. According to the singularity theory, equation (25) is the universal unfolding of normal form $a^{6}+b^{\prime}=0$, the codimension is $2, b^{\prime}$ is the bifurcation parameter, and $\chi_{1}^{\prime}$ and $\chi_{2}^{\prime}$ are the unfolding parameters.

The set of bifurcation points is empty.

The delay point set is calculated as $H_{R}=\left\{\left(3 \chi_{2}^{\prime 2} / \chi_{1}^{\prime 2}\right)-\chi_{2}^{\prime}=\right.$ $0\}$ and $H_{I}=\left\{\chi_{1}^{\prime}\left(d_{2}^{3}-d_{1}^{3}\right)+2 \chi_{2}^{\prime}\left(d_{2}-d_{1}\right)=0\right\}$.

The hyperbolic limit point set is calculated as $D L_{R}=$ $\left\{\left(4 \chi_{2}^{\prime 2} / \chi_{1}^{\prime 2}\right)-\chi_{2}^{\prime}=0\right\}$ and $D L_{I}=\left\{d_{2}^{5}-d_{1}^{5}+4 \chi_{1}^{\prime}\left(d_{2}^{3}-d_{1}^{3}\right)+\chi_{2}^{\prime}\right.$ $\left.\left(d_{2}-d_{1}\right)=0\right\}$.

The migration set is $\Sigma=B \cup H_{R} \cup H_{I} \cup D L_{R} \cup D L_{I}$. The transition set and bifurcation diagrams are shown in Figure 8.
By calculation, the bifurcation point set of the system is an empty set, so the transition set includes the time delay set and hyperbolic limit point set. As shown in Figure 8(a), the transition set divides the plane into 12 regions. In order to facilitate observation, the fine part of the transition set is locally enlarged. The bifurcation diagrams corresponding to each region and the transition set are shown in Figures 8(b) and $8(\mathrm{c})$, and the bifurcation diagrams are abundant. Different bifurcation modes reflect the dynamic behavior of the system without any parameters, so when $d_{1}<a<d_{2}$, the dynamic behavior can be controlled by changing the parameters $\chi_{1^{\prime}}$ and $\chi_{2^{\prime}}$.

(3) When $a>d_{2}$, the bifurcation equation (29) can be obtained from the amplitude frequency curve equation of the system. The calculated values of $\Lambda(a, \omega)$ and $\Phi(a, \omega)$ in Case 3 are shown in equations (27) and (28):

$$
\begin{aligned}
& \qquad \begin{aligned}
\Lambda(a, \omega)= & \left(-\frac{1}{\pi}\left(6 k_{2} \varphi_{1} \cos \varphi_{1}^{2}-5 k_{2} \sin \varphi_{1} \cos \varphi_{1}+3 k_{2} \varphi_{1}-k_{2}\left(\frac{3}{2} \varphi_{1}-\frac{3}{4} \pi+\sin 2 \varphi_{1}+\frac{1}{8} \sin 4 \varphi_{1}\right)\right)\right) a^{3} \\
& +\left(-\frac{1}{\pi}\left(2 k_{3} \varphi_{2} 2+2 k_{1} \varphi_{1}-2 k_{3} \cos \varphi_{2} \sin \varphi_{2}-2 k_{1} \cos \varphi_{1} \sin \varphi_{1}\right)\right) a,
\end{aligned} \\
& \Phi(a, \omega)=\frac{a}{\pi}\left(4 c \omega \varphi_{2}-2 c \omega \sin 2 \varphi_{2}+4 c \omega \varphi_{1}-4 c \omega \varphi_{2}-2 c \omega \sin 2 \varphi_{1}+2 c \omega \sin 2 \varphi_{2}+c \omega \pi-2 c \omega \varphi_{1}+c \omega \sin 2 \varphi_{1}\right) . \\
& \text { The bifurcation equation of the system is as follows: }
\end{aligned}
$$




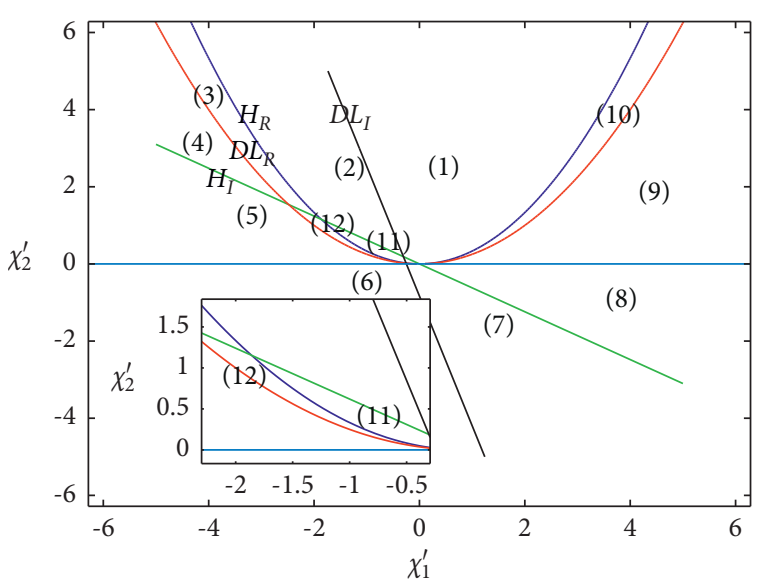

(a)

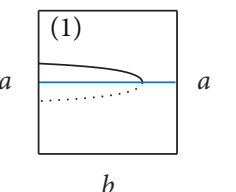

$b$
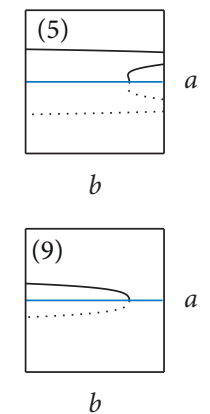

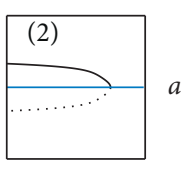

$b$
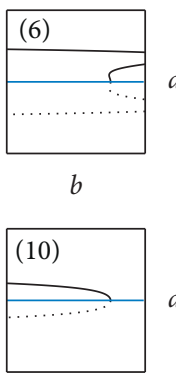

b

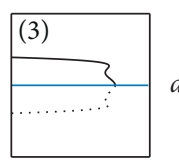

$b$

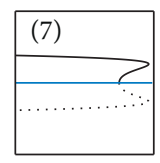

$b$

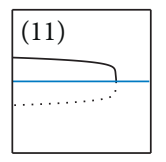

$b$

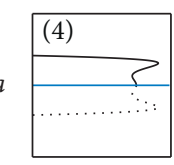

$b$

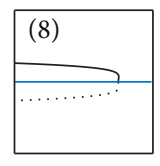

$b$

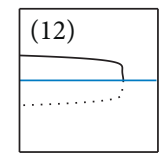

(b)
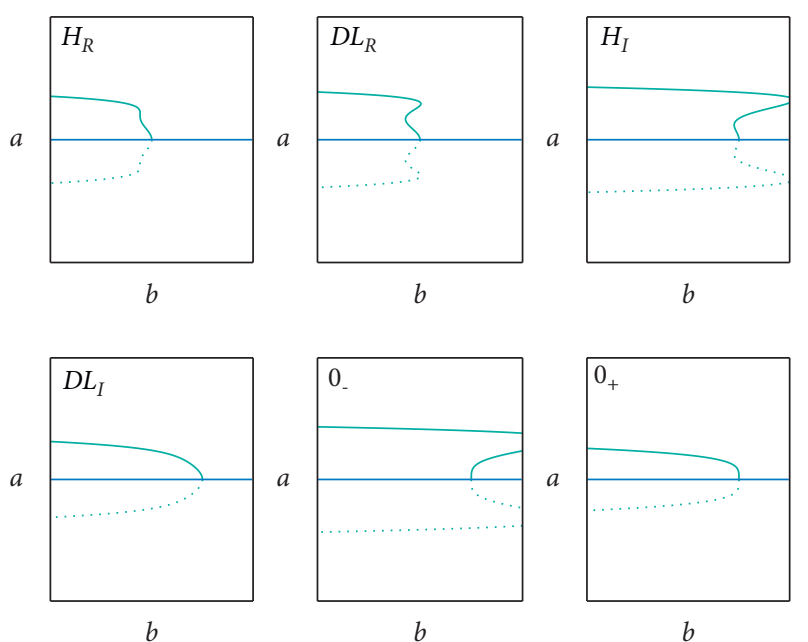

(c)

Figure 8: (a) Transition set and (b, c) bifurcation diagrams of the system.

where $\chi_{1}^{\prime \prime}=\lambda_{1}^{\prime \prime} / \lambda^{\prime \prime}, \chi_{2}^{\prime \prime}=\lambda_{1}^{\prime \prime} / \lambda^{\prime \prime}$, and $b^{\prime \prime}=\lambda_{3}^{\prime \prime} / \lambda^{\prime \prime}$, in which

$$
\begin{aligned}
\lambda^{\prime \prime}= & 0.41 m^{2} \omega^{2}\left(\begin{array}{c}
3 k_{2} \varphi_{1}-4 k_{2}\left(0.375 \varphi_{1}+0.25 \sin 2 \varphi_{1}+0.03 \sin 4 \varphi_{1}-0.59\right) \\
+6 k_{2} \varphi_{1} \cos ^{2} \varphi_{1}-5.0 * k 2 * \cos \varphi_{1} \sin \varphi_{1}
\end{array}\right)^{2}, \\
\lambda_{1}^{\prime \prime}= & 1.27 m \omega\left(\begin{array}{c}
k_{1}-1 m \omega^{2}+0.64 m \omega\left(2 k_{3} \varphi_{2}+2 k_{1} \varphi_{1}-2 k_{1} \cos \varphi_{1} \sin \varphi_{1}-2 k_{3} \cos \varphi_{2} \sin \varphi_{2}\right) \\
+K \omega^{p} \cos \left(\frac{p \pi}{2}-\tau \omega\right)
\end{array}\right) \\
& \left(3 k_{2} \varphi_{1}-4 k_{2}\left(0.375 \varphi_{1}+0.25 \sin 2 \varphi_{1}+0.03125 \sin 4 \varphi_{1}-0.59\right)+6 k_{2} \varphi_{1} \cos ^{2} \varphi_{1}-5 k_{2} \cos \varphi_{1} \sin \varphi_{1}\right), \\
\lambda_{2}^{\prime \prime}= & \left(k 1-m \omega^{2}+0.64 m \omega\left(2 k 3 \varphi_{2}+2 k_{1} \varphi_{1}-2 k_{1} \cos \varphi_{1} \sin \varphi_{1}-2 k_{3} \cos \varphi_{2} \sin \varphi_{2}\right)+K \omega^{p} \cos \left(\frac{p \pi}{2}-\tau \omega\right)\right)^{2}
\end{aligned}
$$




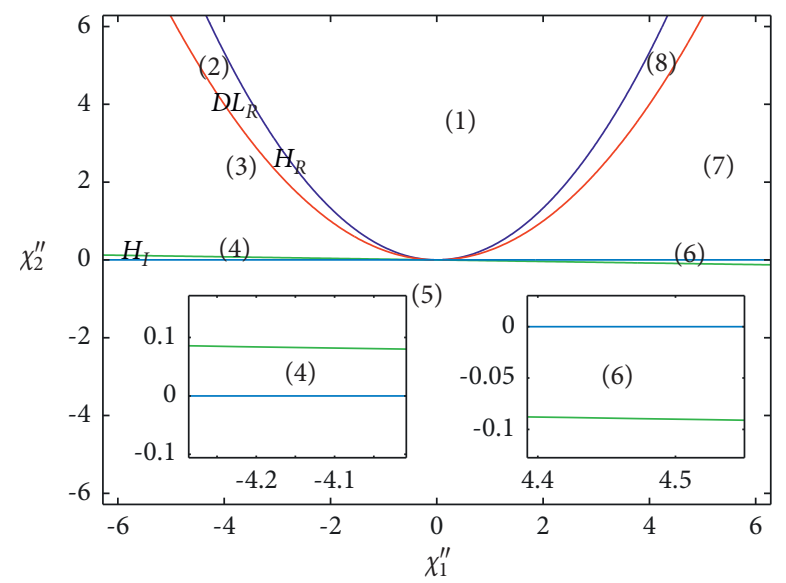

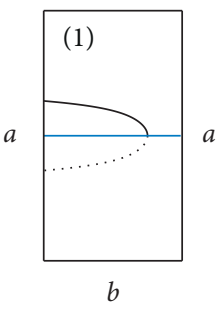
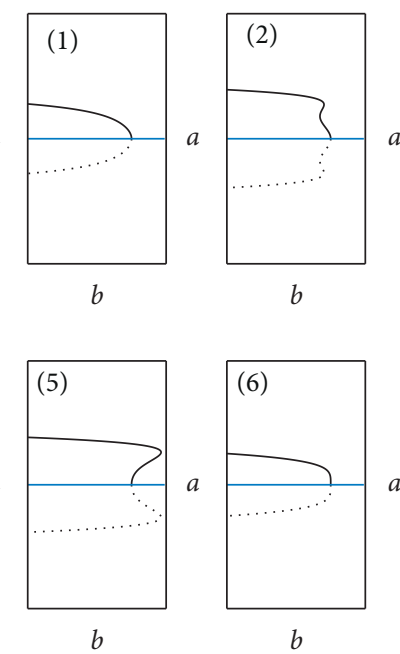

$b$

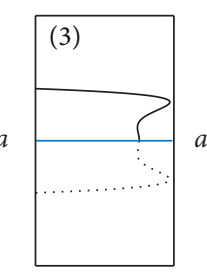

$b$

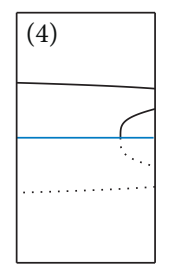

$b$

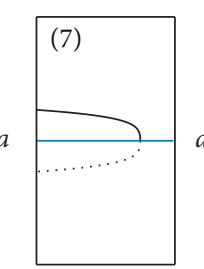

$b$

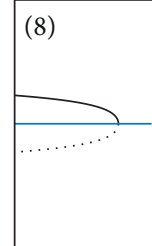

$b$

(a)

(b)

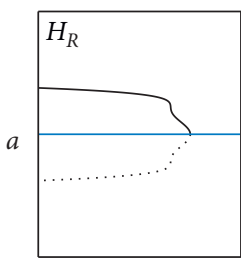

$b$

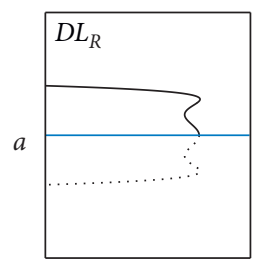

$b$

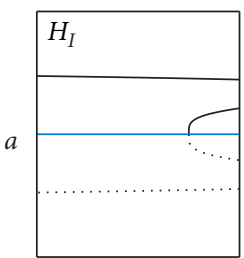

$b$

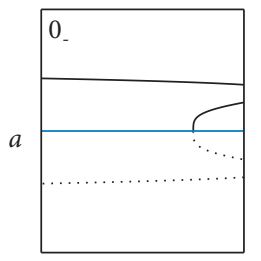

$b$

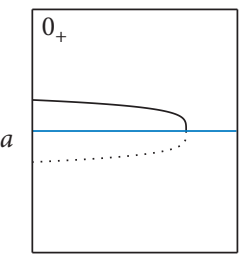

$b$

(c)

Figure 9: (a) Transition set and (b, c) bifurcation diagrams of the system.

$$
\begin{aligned}
& +\left(0.64 m \omega\left(\begin{array}{c}
4 c \omega\left(0.25 \sin 2 \varphi_{1}-0.5 \varphi_{1}+0.79\right)+8 c \omega\left(0.5 \varphi_{2}-0.25 \sin 2 \varphi_{2}\right) \\
+8 c \omega\left(0.5 \varphi_{1}-0.5 \varphi_{2}-0.25 \sin 2 \varphi_{1}+0.25 \sin 2 \varphi_{2}\right)
\end{array}\right)+K \omega^{p} \sin \left(\frac{p \pi}{2}-\tau \omega\right)\right)^{2}, \\
\lambda_{3}^{\prime \prime}= & -F^{2} .
\end{aligned}
$$

In Case 3, the transition set of the system is calculated by using the same form of bifurcation equation with $\chi_{1}^{\prime \prime}$ and $\chi_{2}^{\prime \prime}$ as the unfolding parameters and $b^{\prime \prime}$ as the bifurcation parameters.

The set of bifurcation points is empty.

The delay point set is calculated as $H_{R}=\left\{3 \chi_{2}^{\prime \prime 2} / \chi_{2}^{\prime \prime 2}-\chi_{2}^{\prime \prime}=0\right\}$ and $H_{I}=\left\{\chi_{1}^{\prime \prime} d_{1}^{2}+2 \chi_{2}^{\prime \prime}=0\right\}$.

The hyperbolic limit point set is calculated as $D L_{R}=$ $\left\{4 \chi_{2}^{\prime \prime 2} / \chi_{2}^{\prime \prime 2}-\chi_{2}^{\prime \prime}=0\right\}$ and $D L_{I}$ is an empty set.

The migration set is $\Sigma=B \cup H_{R} \cup H_{I} \cup D L_{R} \cup D L_{I}$. The transition set and bifurcation diagrams are shown in Figure 9.
By calculation, the bifurcation point set of the system is an empty set, so the transition set includes the time delay set and hyperbolic limit point set. As shown in Figure 9(a), the transition set divides the plane into 8 regions. In order to facilitate observation, the fine part of the transition set is locally enlarged. The bifurcation diagrams corresponding to each region and the transition set are shown in Figures 9(b) and $9(\mathrm{c})$, and the bifurcation diagrams are abundant. Different bifurcation modes reflect the dynamic behavior of the system without any parameters, so when $a>d_{2}$, the dynamic behavior can be controlled by changing the parameters $\chi_{1}^{\prime \prime}$ and $\chi_{2}^{\prime \prime}$. 
The bifurcation behavior under different constraints is obtained according to the transfer set of the system in three cases.

\section{Conclusion}

A piecewise nonlinear dynamic model with nonlinear stiffness, damping, and fractional order is proposed for magnetorheological damper. The average method is used to approximate the periodic response of the system. The amplitude frequency response curve of the system is obtained. When the external excitation frequency changes, the amplitude of the system changes, showing a multisolution phenomenon. The difference of amplitude frequency response curves between systems with and without time delay is analyzed and compared. It is concluded that the increase of time delay will increase the response time of the system and change the amplitude frequency response curve of the system. The influence of different time delays on the system is studied. The results show that when the time delay is small, the amplitude of amplitude frequency response curve increases with the increase of time delay. When the time delay is large, the topological structure of amplitude frequency response curve will change. In the second segment, the degree of refraction increases and an independent loop appears in the high frequency region. The influence of the order of fractional differential term on the system is analyzed. With the increase of the order of fractional differential term, the peak value of amplitude frequency response curve decreases gradually. In this paper, the influence of fractional differential coefficients on the system is studied. When the order of the fractional differential term is large, the peak value of the amplitude frequency response curve increases with the increase of the order coefficient of the fractional differential term. When the order of the fractional differential term is larger, the peak value of the amplitude frequency curve decreases with the increase of the order coefficient of the fractional differential term. At the second segment, the degree of refraction decreases or even disappears completely. By using singularity theory, the bifurcation equations of the system varying with the amplitude of external excitation under three kinds of constraints are obtained. With the change of system parameters, the bifurcation topology of the system is given.

\section{Data Availability}

The data used to support the findings of this study are available upon request to the corresponding author (e-mail: mawenlistdu@163.com).

\section{Conflicts of Interest}

The authors declare that they have no conflicts of interest.

\section{Acknowledgments}

This project was supported by the National Natural Science Foundation of China (Nos. 12102273 and 12072205), the Central Government Guided Local Science and Technology
Development Fund Project (No. 206Z1901G), and the S\&T Program of Hebei (No. 20310803D).

\section{References}

[1] Y. Q. Jia and G. P. Jiang, "Synchronization of Fractional Order Chaotic Systems with Time Delay Based on State Observer," Acta Physica Sinica, vol. 16, pp. 26-32, 2017.

[2] J. H. Yang and X. B. Liu, "Resonance Analysis of Periodic Vibration Induced by Linear Time Delay Feedback," Acta physics Sinica, vol. 61, no. 1, pp. 76-83, 2012.

[3] S. F. Wen, Y. J. Shen, and S. P. Shao, "Dynamical analysis of Duffing oscillator with fractional-order feedback with time delay," Acta Physica Sinica, vol. 65, no. 9, pp. 158-167, 2016.

[4] Z. Y. Zhang, X. X. Wang, and S. Z. Lin, "Oscillation and Nonoscillation criteria for Nonlinear Second Order Neutral Delay Differential Equations," Applied Mathematics Letters, vol. 22, no. 7, pp. 1096-1101, 2009.

[5] Q. H. Duan, "A Delayed Predator-Prey Model with Migration Rate and Holling-II Type Functional Response," Advances in Applied Mathematics, vol. 3, no. 4, pp. 231-244, 2014.

[6] X. M. Li, Z. S. Zhao, J. Zhang, and L. K. Sun, "Synchronization of the Coronary Artery System with Input Time-Varying Delay," Chinese Physics B, vol. 25, no. 6, Article ID 060504, 2016.

[7] Q. Q. Chai, "A Method of Identifying Parameters of a TimeVarying Time-Delay Chaotic System," Acta Physica Sinica, vol. 64, p. 24, 2015.

[8] J. H. He, Z. B. Li, and Q. L. Wang, "A New Fractional Derivative and its Application to Explanation of Polar Bear Hairs," Journal of King Saud University Science, vol. 28, no. 2, 2016.

[9] J. H. He, "A Simple Approach to One-Dimensional Convection-Diffusion Equation and its Fractional Modification for E Reaction Arising in Rotating Disk Electrodes," Journal of Electroanalytical Chemistry, vol. 854, 2019.

[10] C. J. LiDY, S. T. Guan, and T. W. Tan, "Research and Implementation of a Fractional Order Predictive Controller," Control theory and Application, vol. 27, no. 5, pp. 658-662, 2010.

[11] Z. Chen, Y. L. Peng, S. W. Wang, and F. L. Yin, "From Discrete to Continuous: Theory, Method and Application of Fractional Order Signal Processing," Acta Electronica Sinica, vol. 40, no. 11, pp. 2282-2289, 2012.

[12] M. Y. Xu and W. C. Tan, "Intermediate Process and Critical Phenomenon: Fractional Operator Theory, Method, Progress and Application in Modern Mechanics," Chinese Science Series G: Physics, mechanics and astronomy, vol. 49, no. 3, pp. 225-238, 2006.

[13] O. Guner, C. K. University, E. A. Sciences, and D. O. I. Trade, "Exp-Function Method and Fractional Complex Transform for Space-Time Fractional KP-BBM Equation," Communications in Theoretical Physics, vol. 68, no. 2, p. 149, 2017.

[14] F. X. Chang Fu-Xuan, W. Chen Jin, and W. Huang, "Anomalous Diffusion and Fractional Advection-Diffusion Equation," Acta Physica Sinica, vol. 54, no. 3, pp. 1113-1117, 2005.

[15] X. J. Liu, L. Hong, and J. Jiang, "Cataclysm in Nonautonomous Fractional Duffing System," Acta Physica Sinica, vol. 65, no. 18, 2016.

[16] X. H. Gu, S. P. Yang, Y. J. Shen, and J. Z. Liu, "Combined Resonance of Fractional Duffing Oscillator," Journal of $\mathrm{Vi}$ bration Engineering, vol. 30, no. 1, pp. 28-32, 2017. 
[17] P. Rahimkhani and Y. Ordokhani, "Numerical Studies for Fractional Pantograph Differential Equations Based on Piecewise Fractional-Order Taylor Function Approximations," Iranian Journal of Science and Technology Transaction A-Science, vol. 42, no. 4, Article ID 442144, 2018.

[18] Y. Geng, M. Song, Y. Lu, and M. Liu, "Convergence and Stability of the Truncated Euler-Maruyama Method for Stochastic Differential Equations with Piecewise Continuous Arguments," Numerical Mathematics: Theory, Methods and Applications, vol. 14, no. 1, pp. 194-218, 2021.

[19] C. I. Martin and B. Basu, "Resonances for Water Waves Over Flows with Piecewise Constant Vorticity," Nonlinear Analysis: Real World Applications, vol. 57, 2021.

[20] O. Ramirez and A. M. Alves, "Bifurcation of Limit Cycles by Perturbing Piecewise Non-Hamiltonian Systems with Nonlinear Switching Manifold," Nonlinear Analysis: Real World Applications, vol. 57, 2021.

[21] F. Liu and H. R. Liu, "Cycle Response Characteristics Research on a Class of Piecewise Nonlinear Elastic and Damping Double Constraint System," Acta Physica Sinica, vol. 64, p. 12, 2015.

[22] J. Yang, L. Q. Zhao, and L. Zhao, "Limit Cycle Bifurcations for Piecewise Smooth Integrable Differential Systems," Discrete \& Continuous Dynamical Systems - B, vol. 22, no. 6, pp. 24172425, 2017.

[23] C. L. Yu, "Limit Cycles for a Class of Discontinuous Generalized Lienard Differential Systems," Advances in Applied Mathematics, vol. 6, no. 1, pp. 20-22, 2017. 